\title{
Research on the Dispersion Stability of Aligned Carbon Nanotubes
}

\author{
Xiao-ning LIANG ${ }^{1, a}$, Wei $\mathrm{LI}^{2, b, \mathrm{c}^{*}}$ \\ ${ }^{1}$ College of Textiles, Donghua University No. 1, Lane 300, Wenhui Rd, Songjiang university town, \\ Shanghai 201620, China \\ ${ }^{2}$ College of Textiles, Donghua University, Shanghai 201620, China \\ a1099006@mail.dhu.edu.cn, ${ }^{b}$ liwei@dhu.edu.cn, cwaiwentougao@outlook.com
}

Keywords: carbon nanotubes, multi-walled, dispersion, stability, aligned.

Abstract. The alignment of carbon nanotubes in composite materials can make the materials anisotropic. This characteristic has drawn a lot of attention recently. This paper examined the stability properties and working lifetime of carbon nanotube suspensions containing highly aligned multi-walled carbon nanotube bundles. Suspensions were made using temperature-controlled and non-temperature-controlled ultrasonic dispersion of aligned carbon nanotubes in solvent. The absorbance of carbon nanotube suspensions was measured employing UV-visible spectrophotometry. The increased stability due to various surfactants (dispersion agents) (SDBS / oleic acid / polymer) was also studied. Results were compared with suspensions made with conventional multi-walled carbon nanotubes.

\section{Introduction}

The superior structure of carbon nanotubes gives them excellent mechanical properties, as well as excellent thermal and electrical conductivity ${ }^{[1-7]}$. They have an extremely wide range of applications in the modern composites industry. Today, in order to further improve performance and extend the range of applications, several types of special-function carbon nanotube materials have been studied. Among them are aligned carbon nanotubes and carbon nanotube bundles ${ }^{[8]}$. The alignment of the carbon nanotubes in materials can make the materials anisotropic, and increase thermal and electrical conductivity in the direction in which the carbon nanotubes are aligned. However, carbon nanotubes do not naturally disperse. It is difficult to get them to disperse evenly within a matrix (polymer / resin) to create a stable system with even particle size distribution.

Current dispersion methods include mechanical dispersion ${ }^{[9]}$, ultrasonic dispersion ${ }^{[10]}$, chemical modification $^{[11]}$, the use of dispersing agents ${ }^{[12]}$, and the polymer wrapping method ${ }^{[13]}$. The use of multiple dispersion methods can often achieve better dispersion results. One effective method is ultrasonic dispersion of carbon nanotubes in solvent to create a stable suspension, followed by mechanically mixing the solution into a resin or other matrix. This method can help improve the quality of products made of carbon nanotube composites. Therefore, creation of a carbon nanotube suspension that remains stable for a reasonable amount of time is a vital part of carbon nanotube composite processing.

Ultrasonic dispersion creates heat and exacerbates the thermal motion of molecules. This leads to secondary agglomeration of carbon nanotubes ${ }^{[14]}$. In addition, chemical and ultrasonic process can destroy the structure of the carbon nanotubes themselves. Mixed dispersion treatments can significantly affect the shape of highly-aligned carbon nanotube bundles. The different morphologies of carbon nanotubes make controlling the dispersion process and the stability of suspensions more complicated.

This study investigated the effects of temperature-control on dispersion stability when utilizing ultrasonic dispersion to make suspensions of highly aligned, multi-walled carbon nanotube bundles in water / acetone solutions. The dispersion stability of conventional multi-walled carbon nanotube suspensions was applied as a reference for comparison. It measure absorption using UV - visible light spectrophotometry. The results showed that our temperature control system could extend the stable, usable lifetime of carbon nanotube suspensions. Aligned carbon nanotubes are more sensitive to 
temperature control than conventional carbon nanotubes. That is, when comparing the stability of suspensions made using ultrasound in temperature-controlled conditions, the research showed that aligned carbon nanotube suspensions were more stable than conventional carbon nanotube suspensions.

\section{Materials and Testing}

Materials. The basic characteristics of the conventional multi-walled carbon nanotubes and the aligned multi-walled carbon nanotubes used in this study can be seen in Table 1. The highly aligned multi-walled carbon nanotubes bunched together to form bundles of tubes that were aligned in the same direction. Bundle length was up to 100 microns ${ }^{[15]}$.

Table 1 Basic Properties of Conventional and Aligned Multi-walled Carbon Nanotubes

Tap Density[g/cm3] Outer Diameter Length [um]

\begin{tabular}{cccc} 
& & {$[\mathrm{nm}]$} & \\
\hline Conventional & 0.18 & $>50$ & $10-20$ \\
Aligned & 0.07 & $10-20$ & $30-100$ \\
\hline
\end{tabular}

Dispersing agents adopted were Sodium Dodecyl Benzene Sulphonate (SDBS), oleic acid, and dispersant polymer (TNEDIS). Types of dispersing agents and corresponding accompanying media are shown in Table 2.

Table 2 Dispersing Agents and Accompanying Media

\begin{tabular}{ccc}
\hline Dispersant Name & Type & Accompanying Medium \\
\hline SDBS & Anionic & Water \\
Oleic Acid & Anionic & Acetone \\
TENDIS & Nonionic / polymer & Acetone \\
\hline
\end{tabular}

Preparation of Suspension Samples. To obtain a carbon nanotube suspension, a $0.5 \mathrm{vol} \%$ carbon nanotube mixture was dispersed in a JAC-2010P model ultrasonic cleaner for 120 minutes.

Suspension Measurements. After the carbon nanotube suspension had sat still for a specified period of time, the top layer of the suspension was took as the sample. A model UV-2550 ultra-violet spectrophotometer measured the absorbance of the sample in light of wavelength of $260.5 \mathrm{~nm}$.

During the test, the absorbance of the sample was nearly proportional to the number of particles per unit volume in the sample ${ }^{[16]}$. Thus, the absorbance could be measured by measuring the dispersion level of carbon nanotubes in the suspension. Dispersion level mainly includes: size of particles in the suspension; evenness of particle distribution within the suspension; and sedimentation characteristics of the particles in the suspension (stability of the suspension).

Generally speaking, the smaller the particles, the easier they are suspended in the upper part of the suspension. Bigger particles tend to sink to the bottom layers of the suspension more quickly. Solid particles are influenced by dispersing agents and gravity. The heavier the particles, the easier they sink. More effective dispersing agents are better in countering this sinking effect.

\section{Results and Discussions}

The Relationship between Sitting Time and Absorbance. Figure 1 shows the absorbance of SDBS/water suspension samples containing each type of carbon nanotubes, after sitting for various periods of time. The figure shows that the absorbance of both types of solution dropped quickly at first. The rate slowed as time moves on, and the curves flatten out. The absorbance of the suspension containing conventional nanotubes dropped in a more stable fashion. After 4 hours, it had reached a relatively stable state. After that, absorbance changed very little. The absorbance of the suspension 
containing aligned carbon nanotubes dropped very quickly and unevenly at first. After four hours, its slow decline rate was slower than that of the conventional carbon nanotube suspension. Also, it reached the slow, stable stage sooner. This was mainly because there was a larger range of particle sizes in the aligned carbon nanotube suspension. Changes in the morphology during dispersion are more complicated, and the range of particle sizes in the suspension is larger. Larger particles sink quickly while the suspension is sitting. This caused the absorbance to decrease quickly at first. However, aligned carbon nanotubes are less dense than regular carbon nanotubes. So, as time goes on, absorbance decreased at a slower rate than it did for the conventional carbon nanotube suspension. Also, aligned carbon nanotubes are more sensitive to temperature. That is, changes in temperature have larger effects on the dispersion level. Suspensions made in non-temperature-controlled ultrasonic processes slowly cool to room temperature when placed in room temperature environments. This was another reason why, during the cooling period, the absorption rate of aligned nanotube suspensions dropped faster than it did for conventional carbon nanotube suspensions.

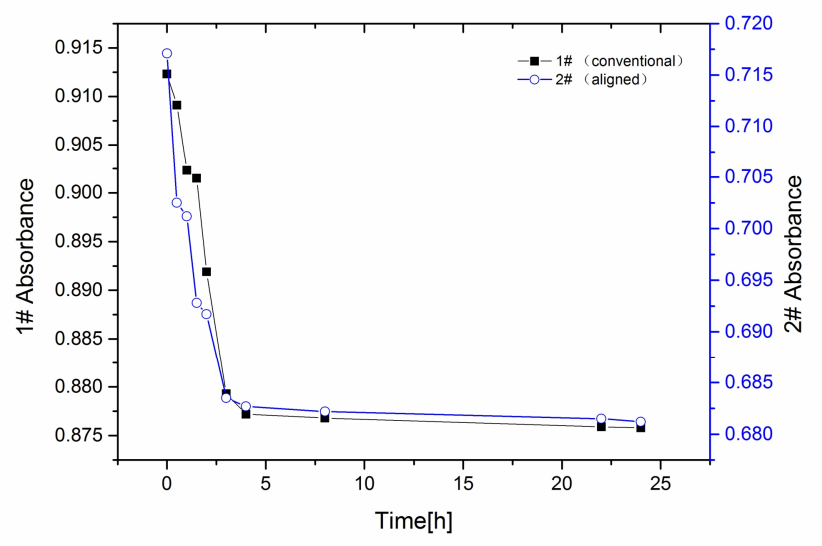

Figure 1 Relationship between Sitting Time and Absorbance

The Effect of Temperature Control on Suspension Stability. Figure 2 shows how the absorbance of aligned carbon nanotube suspensions made using temperature-controlled and non-temperature-controlled processes changed over time. The figure shows that the suspension made using a temperature-controlled process had high initial absorbance, which indicated a good dispersion. During the first two hours, the absorbance of the temperature-controlled suspension dropped more slowly than that of the non-temperature-controlled suspension. Also, the overall drop in absorbance during the first two hours was less for the temperature-controlled suspension than it was for the non-temperature-controlled suspension. During the ultrasonic dispersion process, the temperature of the suspension rose from $20{ }^{\circ} \mathrm{C}$ to as high as $50{ }^{\circ} \mathrm{C}$ due to heat caused by the ultrasonic treatment process. High temperatures cause intense thermal motion of molecules. This means that collisions between carbon nanotube molecules can more easily result in secondary agglomeration. These larger agglomerations sink more easily. Agglomerations do not occur as easily during temperature-controlled dispersion, so temperature-controlled suspensions are more stable. 


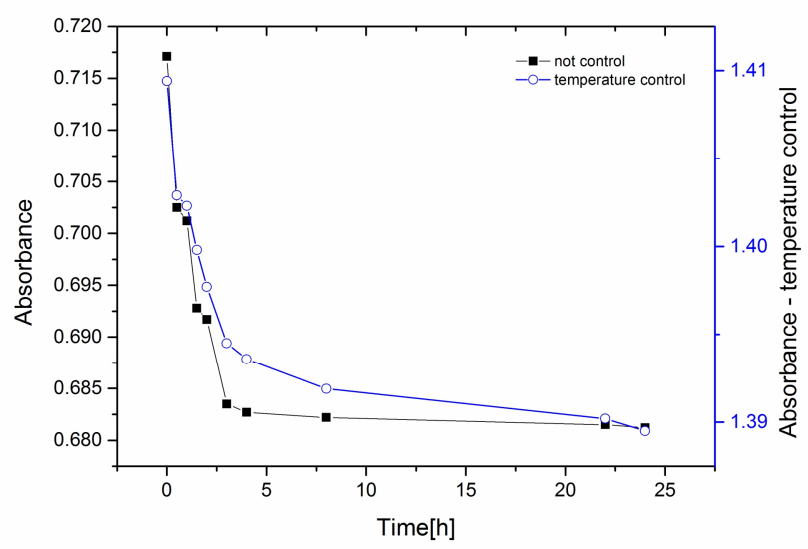

Figure 2 The Effects of Temperature-Controlled Dispersion on the Stability of Aligned Carbon Nanotube Suspensions

Figure 3 shows the absorbance of aligned and conventional carbon nanotube suspensions made using temperature-controlled ultrasonic dispersion processes as functions of sitting time. The figure shows that in the first two hours, the absorbance of the aligned carbon nanotube suspension decreased faster and stabilized sooner. The change in overall absorbance of the aligned carbon nanotube suspension was less than the conventional carbon nanotube suspension, and it decreased at variable rates, while the conventional nanotube suspension decreased more smoothly. This is because aligned carbon nanotubes are more sensitive to changes in temperature. Also, the particle sizes in the aligned carbon nanotube suspension were less uniform, and shapes of agglomerations made of very thin diameter nanotubes were more complex.

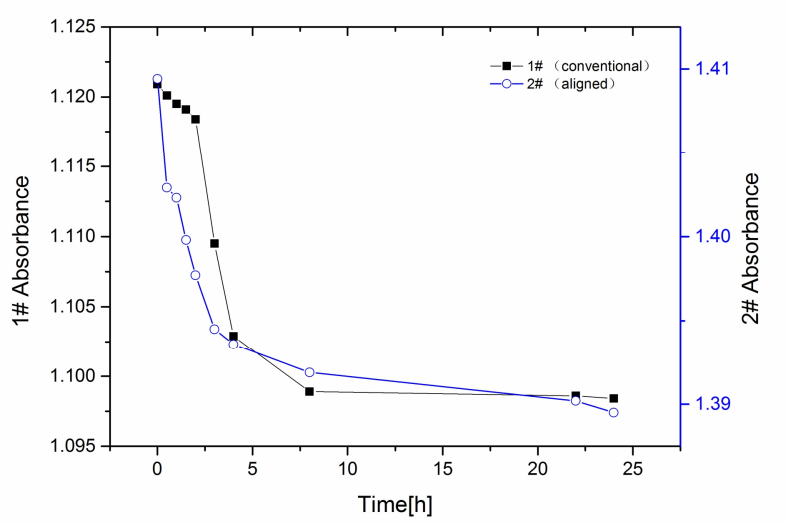

Figure 3 Absorbance of Suspensions Made in Temperature-Controlled Processes as Functions of Sitting Time

Overall, temperature-control was beneficial to the dispersion performance of both types of carbon nanotubes. It effectively eliminated or mitigated the negative effects caused by increased molecular motion (and agglomeration) at high temperatures during the ultrasonic dispersion process. However, the extent of the benefit depended upon the material. Temperature control was especially beneficial to the stability of suspensions made of aligned carbon nanotubes, which were more sensitive to temperature variations. In addition, judging by the initial rate of decrease of absorbance, temperature control made the dispersion process more stable and increased the overall effectiveness of the dispersion process.

The Effects of Dispersants upon the Stability of Suspensions. SDBS, oleic acid, and TENDIS were tested separately to disperse each type of carbon nanotube suspension. Each type of dispersant was 
employed according to its optimal performance parameters. Usable lifetime (time for absorbance to decrease 5\%) for each combination is as shown in Figure 4.

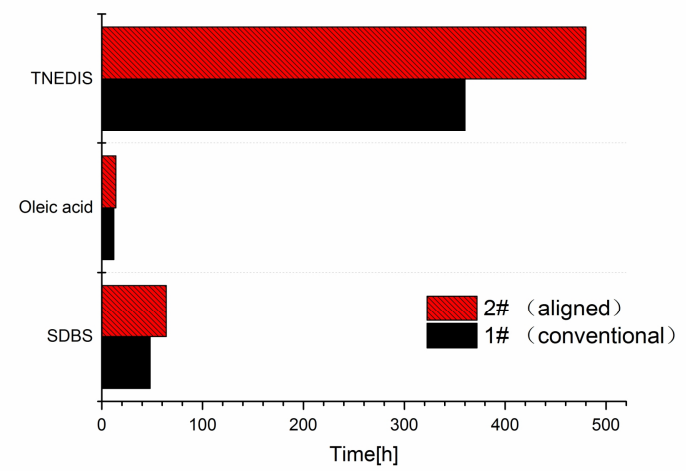

Figure 4 Usable lifetime of Carbon Nanotube Suspensions Using Three Types of Dispersing Agents Under Ideal Conditions.

Figure 4 shows that suspensions dispersed by dispersant polymer TNEDIS could maintain dispersion quality for the longest period of time. It gives the suspensions the longest usable lifetime. The conventional carbon nanotube suspension with TNEDIS dispersing agent had a usable lifetime of $360 \mathrm{~h}$, and the aligned carbon nanotube suspension with TNEDIS had a usable lifetime of $480 \mathrm{~h}$. SDBS dispersing agent yielded the second longest usable lifetime. Oleic acid was the least effective dispersing agent. The type of carbon nanotube in the suspension had a large effect on usable time period. Suspensions made utilizing aligned carbon nanotubes always had longer usable lifetimes than those with conventional carbon nanotubes. This is because aligned carbon nanotubes have smaller density and do not sink as easily as conventional carbon nanotubes. Also, the special shape of aligned carbon nanotubes causes them to line up parallel to each other, making the carbon nanotubes relatively straight after dispersion. They do not easily become intertwined and form agglomerations. Therefore, they do sink easily.

The Effects of Shaking on Usable lifetime. Carbon nanotube suspensions are always shaken before use. The effect of shaking on the usable lifetime of carbon nanotube suspensions is shown in Figure 5.

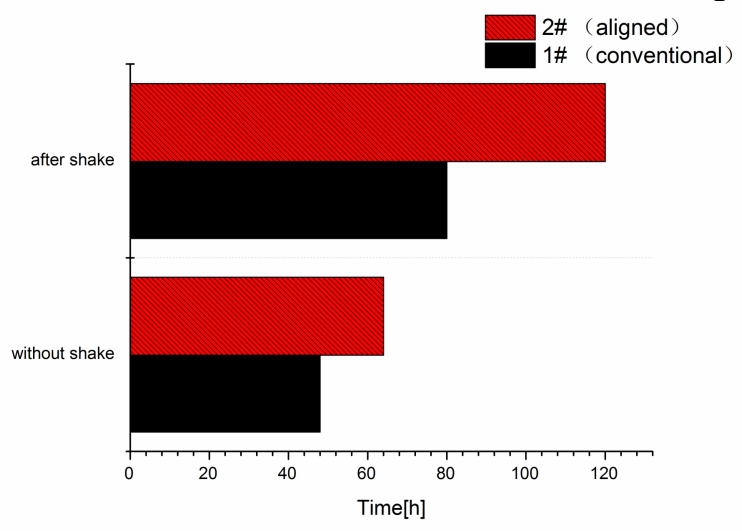

Figure 5 The Effect of Shaking on Usable Lifetime

Figure 5 shows that simply shaking a carbon nanotube suspension could extend its usable lifetime. This is mainly because a considerable part of the agglomerations formed between carbon nanotubes in suspension are soft agglomerations. The agglomerations are easily broken down and the molecules re-dispersed by shaking. These agglomerations do not have much effect on the quality of the carbon nanotube suspension. As long as it is shaken before use, it is OK. 


\section{Conclusions}

This article applied water bath ultrasound to disperse aligned carbon nanotubes and conventional carbon nanotubes, and studied the stability of the resulting suspensions. The major conclusions are:

(1) Under non-temperature-controlled conditions, aligned carbon nanotube suspensions were less stable than conventional carbon nanotube suspensions.

(2) Temperature-controlled ultrasound dispersion resulted in suspensions with better dispersion level and stability versus non-temperature-controlled dispersion. Aligned carbon nanotube suspensions were more sensitive to temperature changes than conventional carbon nanotube suspensions.

(3) Temperature-controlled ultrasound suspensions containing aligned carbon nanotubes were more stable than those containing conventional carbon nanotubes. Dispersing agent effectiveness was TNEDIS > SDBS > oleic acid.

\section{References}

[1] M.M.J. Treacy, T.W. Ebbesen, J.M. Gibson: Exceptionally high Young's modulus observed for individual carbon nanotubes. Nature,381 (1996) 678-80.

[2] Y. Yosida: High-temperature shrinkage of single-walled carbon nanotube bundles up to $1600 \mathrm{~K}$. J ApplPhys, 87 (2000)3338-41.

[3] S. Berber, Y.K. Kwon, D. Toma'nek: Unusually high thermal conductivity of carbon nanotubes. Phys Rev Lett, 84 (2000) 4613-6.

[4] J. H. Du, J. Bai, H. M. Cheng: Express. Polym.Lett.,1 (2007)253- 265.

[5] D.A. Heller, R. M. Mayrhofer, S. Baik et al: J. Am. Chem. Soc., 126 (2004)14567-14573.

[6] G.T. Liu, Y.C. Zhao, K.H. Zheng et al: Nano. Lett.,9 (2009)239-244.

[7] T. Tanaka, H.H. Jin, Y. Miyata et al: Nano. Lett.,9 (2009)1497-1500.

[8] A. Peigney, C. Laurent, E. Flahaut, R.R. Bacsa, A. Rousset: Specific surface area of carbon nanotubes and bundles of carbon nanotubes. Carbon,39 (2001)507-14.

[9] S.J. Yoo, S.H. Han, W.J. Kim: A combination of ball milling and high-ratio differential speed rolling for synthesizing carbon nanotube/copper composites. Carbon,61(2013) 487-500

[10] K.J. Ziegler, Z.J. Gu, Z. Chen, et al: Nano Techn, 16 (2005) 539

[11] E. C.-W. Ou, L.B. Hu, G.C.R. Raymondt, et al: ACS Nano,3 (2009) 2258-2264.

[12] K. Yurekli, C.A. Mitchell, R. Krishnamoorti. J Am ChemSoc;126 (2004) 9902-3.

[13] C. Park, Z. Ounaies, K.A. Watson: Dispersion of single wall carbon nanotubes by in situ polymerization under sonication [J].ChemPhys Lett, 364 (2002) 303

[14] C. Lu, Z. Zhang, J. Zhou, S. Zhang: Influence of Different Dispersants on the Dispersion Stabilities of Nickel Nanopowders in Ethanol. Materials Review,5 (2007) 165-167

[15] X. -n. Liang and W. Li, J. Dispers. Sci. Technol. (2015), DOI:10.1080/01932691.2015.1101608.

[16] C. Wang, Z. Zhang, C. Jang: Effect of different dispersants on dispersion capability of nano-sized iron powders.Foundry Technology,5 (2007) 614-617 